\title{
Necrotizing Enterocolitis in Multi Fetal Pregnancies: Can We Find a Key in Placental Abnormalities? A Retrospective Data Analysis
}

\author{
Rosan R. Aapkes ${ }^{1}$, Karien E. A. Hack ${ }^{2}$, Corine Koopman-Esseboom ${ }^{3}$, Peter G. J. Nikkels ${ }^{4}$, \\ Jan B. Derks ${ }^{1}$, Hens A. A. Brouwers ${ }^{3}$ \\ ${ }^{1}$ Department of Obstetrics, University Medical Center Utrecht (UMCU), Utrecht, The Netherlands \\ ${ }^{2}$ Department of Gynecology and Obstetrics, Gelre Ziekenhuizen, Apeldoorn, The Netherlands \\ ${ }^{3}$ Department of Neonatology, University Medical Center Utrecht (UMCU), Utrecht, The Netherlands \\ ${ }^{4}$ Department of Pathology, University Medical Center Utrecht (UMCU), Utrecht, The Netherlands \\ Email: *r.r.aapkes@gmail.com
}

How to cite this paper: Aapkes, R.R., Hack, K.E.A., Koopman-Esseboom, C., Nikkels, P.G.J., Derks, J.B. and Brouwers, H.A.A. (2019) Necrotizing Enterocolitis in Multi Fetal Pregnancies: Can We Find a Key in Placental Abnormalities? A Retrospective Data Analysis. Open Journal of Obstetrics and Gynecology, 9, 1611-1623. https://doi.org/10.4236/ojog.2019.912157

Received: November 5, 2019

Accepted: December 28, 2019

Published: December 31, 2019

Copyright $\odot 2019$ by author(s) and Scientific Research Publishing Inc. This work is licensed under the Creative Commons Attribution International License (CC BY 4.0).

http://creativecommons.org/licenses/by/4.0/ (c) (i) Open Access

\begin{abstract}
Objective: We aimed to evaluate the relationship between chorionicity, placental abnormalities and necrotizing enterocolitis in multiple pregnancies. We hypothesized that unbalanced interfetal transfusion through vascular anastomoses in monochorionic placentation causes hypoperfusion of the intestinal mucosa, increasing the risk of developing necrotizing enterocolitis. Material and methods: All women with multiple pregnancies who delivered at the University Medical Center Utrecht between January 1995 and December 2015 were retrospectively selected. We compared baseline characteristics and neonatal and maternal outcomes. Secondly, we analyzed ultrasound and placental pathology findings of monochorionic multiples with and without necrotizing enterocolitis. Finally, we compared illness characteristics of necrotizing enterocolitis in monochorionic multiples with necrotizing enterocolitis in dichorionic multiples. Results: We included 2859 dichorionic and 817 monochorionic neonates. Necrotizing enterocolitis occurred significantly more often in monochorionic as compared to dichorionic neonates $(3.3 \%$ and $1.6 \%$ respectively), also after correction for birthweight, gestational age and nulliparity (OR 1.7, 95\% CI 1.0 - 2.8). Ultrasound abnormalities were not associated with necrotizing enterocolitis. Histopathology showed that necrotizing enterocolitis was significantly associated with the presence of unbalanced interfetal transfusion (76.9\% of monochorionic with necrotizing enterocolitis versus $31.4 \%$ of cases without necrotizing enterocolitis, $P=0.001)$. Conclusion: Necrotizing enterocolitis is more common in monochorionic multiples as compared to dichorionic multiples, at least in part due to the presence of and related to the presence of unbalanced interfetal transfusion through ar-
\end{abstract}


terial-venous anastomoses in the placenta. Possibly, subtle ischemic damage caused by intra-uterine fetal hypotension or anemia plays a key role in the development of necrotizing enterocolitis in monochorionic twins.

\section{Keywords}

NEC, Placenta, Multi Fetal Pregnancy, Twin Pregnancy

\section{Introduction}

Necrotizing enterocolitis (NEC) is the most prevalent gastrointestinal illness in neonates, affecting $1.0 \%-7.7 \%$ of infants admitted to a neonatal intensive care unit (NICU) [1] [2]. Preterm birth and low birthweight are well-known risk factors for the development of NEC [3], and congenital heart disease, anemia and formula feeding (versus breast feeding) also seem to increase the risk to develop NEC [4]. Its pathophysiology remains poorly understood and appears to be multifactorial, with an important role for ischemic necrosis of the intestinal mucosa [5] [6] [7]. A higher incidence of NEC in multiple pregnancies has been described, most notably in monochorionic (MC) pregnancies [8] [9]. This increased risk of NEC in MC twin pregnancies compared with dichorionic (DC) twin pregnancies remains after correction for gestational age and birthweight [9]. Differences in morphologic characteristics between MC and DC placentas, especially the presence of placental vascular anastomoses with its related risk of interfetal transfusion, may explain the higher rate of NEC in MC twins.

We hypothesized that interfetal transfusion through vascular anastomoses in the MC placenta may lead to a hemodynamic imbalance. This imbalance could cause hypoperfusion of end organs such as the intestinal mucosa, resulting in subtle hypoxic damage and an increased risk of developing NEC after birth. The objective of the current study was to evaluate the relationship between chorionicity, placental abnormalities and NEC.

\section{Materials and Methods}

The study was approved by the research ethics committee of our institution. Medical records of all multiple pregnancies (twins and triplets) delivered at our hospital, a tertial referral hospital, between January 1995 and December 2015 were retrieved from the electronic database. Women who had their prenatal care at our institution as well as women referred during their pregnancy who delivered at the institution were included. We excluded neonates with a gestational age of less than 25 weeks and pregnancies complicated by intra-uterine fetal death. Twin-to-twin transfusion syndrome (TTTS) cases are included in analysis. Cases with unknown chorionicity were excluded. All other cases were included.

Baseline characteristics and neonatal and maternal outcomes were documented 
according to chorionicity. Chorionicity was determined based on placental pathology findings after birth. Monochorionic monoamniotic twins were included in the MC group. In case of triplets, they were classified as follows: offspring of a trichorionic triamniotic triplet pregnancy were included in the DC group, and offspring of a monochorionic triplet (either triamniotic, diamniotic or monoamniotic) were included in the $\mathrm{MC}$ group. In case of dichorionicity of the triplet the singleton was included in the DC group whereas both fetuses with MC placentation (either diamniotic or monoamniotic) were included in the MC group. Standard multiple pregnancy monitoring consisted of a first trimester ultrasound with determination of chorionicity, a detailed anomaly scan at 20 weeks of gestation and regular ultrasound assessment of growth in DC multiple pregnancies as well as every two weeks ultrasound assessment of growth, amniotic fluid and Doppler measurements in MC multiple pregnancies. Elective delivery of uncomplicated MC multiple pregnancies was planned around 37 weeks. In uncomplicated DC pregnancies, a spontaneous onset of labor was awaited. If a growth discordancy of $>20 \%$ was found in DC twins, delivery was pursued around 38 weeks. In case of triplet pregnancies, the timing of delivery was individualized after 34 weeks of gestation.

Stillbirth was defined as intra-uterine demise of a fetus weighing $>500$ gram and/or with $\geq 22$ completed weeks of gestation. Early neonatal mortality was defined as death of an infant during the first 7 days of life and late neonatal mortality as death between 8 and 28 days after birth. Intra-uterine growth restriction (IUGR) was defined as an estimated fetal weight below the $10^{\text {th }}$ percentile with abnormal Doppler flow patterns of the umbilical artery.

Admission to a NICU as well as duration of the NICU stay was recorded. Apgar scores and umbilical cord blood samplings were collected to identify cases of perinatal asphyxia (an apgar after 5 minutes $\leq 7$ and/or an $\mathrm{pH}$ of the umbilical cord of $<7.10)$. Severe neonatal morbidity was defined as the presence of respiratory distress syndrome (RDS), intraventricular hemorrhage (IVH), sepsis, and/or NEC. The composite neonatal morbidity score is positive, when at least one of these morbidities was present. Characteristics associated with the onset of NEC were recorded, including systemic signs (temperature instability, apnea, bradycardia, lethargy), abdominal symptoms (gastric retention, abdominal distention, emesis, haem positive stool) and radiographic signs (intestinal dilatation, ileus, intestinal pneumatosis. NEC was diagnosed according to the modified Bell's criteria [10]. When NEC was suspected, either surgery or conservative management including supportive care and antibiotics was applied, depending on its severity.

First, we analyzed the occurrence and characteristics of NEC after MC and DC multiple pregnancies. The age at diagnosis, duration, symptoms, results of blood tests, need for surgical intervention and pathology findings after surgery were compared between MC and DC multiples.

The cases of MC multiples complicated by NEC were then analyzed in greater 
detail. Ultrasound findings, especially Doppler assessments of the umbilical artery, and placental pathology reports were evaluated.

Placental weight, distribution of the different placental territories, type of cord insertion (central, paracentral, marginal or velamentous) and number, type and size of vascular anastomoses were recorded. Anastomoses could be arterial-arterial (AA), venous-venous (VV), arterial-venous (AV) or venous-arterial (VA). The size (in millimeters) of the anastomoses was measured. Unbalanced interfetal transfusion was defined as a net caliber difference between the AV and VA anastomoses between both placental territories. Unequal placental sharing was defined as one twin receiving blood from $>60 \%$ of the placenta. The methods of the placental pathology studies have previously been described in detail [11].

Statistical analysis was performed using SPSS software, version 22.0. Differences in categorical variables were assessed using the Chi square test. Continuous variables were analyzed using the independent sample t-test or the Mann-Whitney $U$ test. The possible association between different variables and the development of NEC in MC and DC multiples was assessed utilizing logistic regression analyses, correcting for possible confounders. All the significantly different variabeles between MC and DC were analyzed. $P$-values $<0.05$ were considered significant.

\section{Results}

During the study period, 1783 twins and 37 triplets were born at our institution that met the inclusion criteria, resulting in 2859 neonates with DC placentation and 817 with MC placentation (44 of them monochorionic monoamniotic twins). Baseline characteristics are shown in Table 1. Women in the DC group were on average older and they were nulliparous and pregnant after spontaneous conception more often as compared to women with a MC multiple pregnancy. A higher proportion of MC neonates was admitted to a NICU, and they needed NICU care longer than DC neonates. The rate of neonatal morbidity was higher in the MC group, with NEC being significantly more common in MC twins as compared to DC twins (3.3\% versus $1.6 \%, P=0.003$ ).

Univariate logistic regression analysis showed that chorionicity, birthweight and nulliparity were significantly associated with NEC. After adjusting for these potential confounders, NEC remained more prevalent in MC neonates as compared to DC multiples (OR 1.7, 95\% CI 1.0 - 2.8; Table 2).

MC neonates with NEC were born with a shorter gestational age and a subsequently lower birthweight than MC neonates without NEC (Table 3). Twin-to-twin transfusion syndrome (TTTS) was found in $29.6 \%$ of MC multiples that later developed NEC, and in $11.5 \%$ of infants without NEC $(P=0.005)$. Eight neonates developed NEC after a pregnancy complicated by TTTS, four of which were the donor twins and the other four were recipients. Hemoglobin levels were lower and blood transfusions given more often in infants developing NEC compared with infants without NEC; $72.7 \%$ of patients with NEC needed a blood 
Table 1. Characteristics of neonates born after a multiple pregnancy in our institution between 1995 and 2015.

\begin{tabular}{cccc}
\hline & DC (n= 2859) & MC (n= 817) & $P$-value \\
\hline Maternal age (years), mean (SD) & $32.56(5.1)$ & $31.28(5.0)$ & $<0.001$ \\
Spontaneous conception, n (\%) & $1455(50.9)$ & $688(84.4)$ & $<0.001$ \\
Nulliparity, n (\%) & $1622(56.7)$ & $407(49.8)$ & $<0.001$ \\
Gestational age at delivery (weeks), mean (SD) & $35.2(7.1)$ & $34.1(3.4)$ & $<0.001$ \\
Sex distribution, male, n (\%) & $1464(51.2)$ & $399(48.8)$ & 0.232 \\
Birthweight (grams), mean (SD) & $2246.5(753.0)$ & $2039.2(677.4)$ & $<0.001$ \\
Birthweight < p10, n (\%) & $715(25.1)$ & $165(20.3)$ & 0.005 \\
Apgar score $\leq 7$ at 5 minutes, n (\%) & $267(9.4)$ & $105(15.0)$ & 0.003 \\
Umbilical artery pH < 7.10, n (\%) & $1048(36.7)$ & $321(39.5)$ & 0.159 \\
Composite neonatal morbidity, n (\%) & $573(20.1)$ & $200(24.5)$ & 0.006 \\
- NEC, n (\%) & $47(1.6)$ & $27(3.3)$ & 0.003 \\
- IVH, n (\%) & $168(5.9)$ & $57(7.0)$ & 0.448 \\
- Sepsis, n (\%) & $242(8.4)$ & $92(11.3)$ & 0.015 \\
- RDS, n (\%) & $396(13.8)$ & $137(16.8)$ & 0.038 \\
- TTS & & $99(12.1)$ & \\
Admission to NICU, n (\%) & $1018(35.6)$ & $407(49.8)$ & $<0.001$ \\
Early neonatal death, n (\%) & $6.14(14.6)$ & $37.11(32.0)$ & $<0.001$ \\
Late neonatal death, n (\%) & $93(3.3)$ & $39(4.8)$ & 0.039 \\
Length of stay in NICU (days), median (SD) & $20(0.7)$ & $6(0.7)$ & 0.917 \\
\hline
\end{tabular}

${ }^{\dagger}$ Neonate of a twin pair was excluded because of missing data.

Table 2. Regression analysis univariate analysis of the association of different variables and NEC.

\begin{tabular}{cccc}
\hline Variable & $P$-value & $\begin{array}{c}\text { OR } \\
(95 \% \text { confidence interval) }\end{array}$ & $\begin{array}{c}\text { OR adjusted }^{\dagger} \\
\text { (95\% confidence interval) }\end{array}$ \\
\hline Monochorionic placentation & 0.004 & $2.043(1.264-3.300)$ & $1.666(1.010-2.745)^{\star}$ \\
Birth weight (grams) & $<0.001$ & $0.998(0.997-0.999)$ & $0.998(0.998-0.999)^{\star *}$ \\
Nulliparity & 0.032 & $1.711(1.047-2.796)$ & $1.009(0.602-1.690)$ \\
Gestational age (weeks) & 0.638 & $0.994(0.970-1.019)$ \\
Sex distribution, male & 0.555 & $0.870(0.549-1.380)$ \\
Age mother (years) & 0.256 & $0.976(0.936-1.018)$ \\
\hline
\end{tabular}

${ }^{\dagger}$ Adjusted for monochorionic placentation, birth weight, nulliparity, ${ }^{\star} P=0.045,{ }^{* *} P=0.000$.

Table 3. (a) Characteristics of neonates with and without NEC born after a MC multiple pregnancy in our institution between 1995 and 2015; (b) Characteristics of neonates born after a MC pregnancy in our institution between 2006 and 2015.

(a)

\begin{tabular}{cccc}
\hline Variable & NEC $(\mathrm{n}=27)$ & No NEC $(\mathrm{n}=790)$ & $P$-value \\
\hline Maternal age (years), mean (SD) & $31.7(6.1)$ & $31.3(5.0)$ & 0.656 \\
Nulliparity, n (\%) & $19(70.4)$ & $388(49.1)$ & 0.030 \\
\hline
\end{tabular}


Continued

\begin{tabular}{cccc}
\hline Gestation in weeks, mean (SD) & $30.8(3.6)$ & $34.2(4.7)$ & 0.001 \\
Birthweight, mean (SD) & $1228.1(498.7)$ & $2067.1(665.5)$ & $<0.001$ \\
Birthweight < p10, n (\%) & $9(33.3)$ & $156(19.9)$ & 0.088 \\
Sex distribution, male, n (\%) & $10(37.0)$ & $389(48.2)$ & 0.212 \\
TTTS, n (\%) & $8(29.6)$ & $91(11.5)$ & 0.005 \\
\hline
\end{tabular}

(b)

\begin{tabular}{cccc}
\hline Variable & NEC $(\mathrm{n}=11)$ & No NEC $(\mathrm{n}=494)$ & $P$-value \\
\hline Blood transfusion $<$ 7 days post-partum, n $(\%)^{\dagger}$ & $8(72.7)^{\ddagger}$ & $63(12.8)$ & $<0.001$ \\
Blood transfusion during hospitalization, n (\%) & $9(81.8)$ & $91(18.5)$ & $<0.001$ \\
Hb post-partum, mean (SD)* & $9.7(2.3)$ & $10.8(2.1)$ & 0.087 \\
Hb difference between both infants, mean (SD)* & $1.7(1.1)$ & $2.1(2.0)$ & 0.884 \\
$\mathrm{Hb}<8.0 \mathrm{mmol} / \mathrm{L}, \mathrm{n}(\%)$ & $2(18.2)$ & $174(35.3)$ & 0.265 \\
\hline
\end{tabular}

2006-2015 $508 \mathrm{MC}$ neonates (twins and triplets), ${ }^{\dagger} 131$ missing data. ${ }^{*}$ All of the neonates with NEC and a blood transfusion $<7$ days post-partum are donor neonates.

transfusion within 7 days postpartum, versus $12.8 \%$ of patients without NEC ( $P$ $<0.000)$.

Ultrasound data are available from 2006 to 2015 ( $n=436$ after exclusion of missing data), especially Doppler studies of the umbilical artery. Placenta pathology data are available from 1998 to 2015 ( $n=398$ after exclusion of missing data). Table 4 shows the ultrasound findings in the MC multiples with and without NEC. There were no significant differences in the presence of Doppler abnormalities of the umbilical artery or the middle cerebral artery, nor was there a significant difference in the occurrence of IUGR.

An overview of the placental findings in MC multiple pregnancies with and without NEC is shown in Table 5 . Placental weight was significantly lower in neonates developing NEC. There were no differences in the incidence of unequal placental sharing and types of umbilical cord insertions. The presence of any kind of vascular anastomosis was not significantly different between cases with and without NEC. In neonates with NEC, there is a trend towards less AA-anastomoses compared to neonates without NEC (69.2\% and $85.2 \%$ respectively, $P=0.116)$. Unbalanced interfetal transfusion through $\mathrm{AV}$-anastomoses between both placental territories was more common in the NEC group (76.9\% versus $31.4 \%, P=$ $0.001)$. In the majority of cases with unbalanced interfetal transfusion, the donor twin developed NEC.

The presentation and course of NEC did not differ between DC and MC multiples, as can be seen in Table 6. In the DC group, the first onset of NEC was seen 11 days after birth as compared to day 15 postpartum in the MC group ( $P=$ 0.190). Clinical, biochemical (including highest CRP value, lowest platelet level, highest and lowest white blood count level) and radiologic signs did not differ between both groups. However, a trend toward a higher proportion of MC multiples needing surgery as compared to DC multiples ( $72.7 \%$ vs $46.2 \%, P=0.138)$ was seen. 
Table 4. Ultrasound findings of monochorionic multiples with and without NEC in our institution between 2006 and 2015 .

\begin{tabular}{cccc}
\hline Variable & NEC (n= 9) & No NEC (n= 427) & $P$-value \\
\hline $\begin{array}{c}\text { At least one abnormal Doppler finding of the } \\
\text { umbilical artery during pregnancy, n (\%) }\end{array}$ & $2(22.2)$ & $53(12.4)$ & 0.317 \\
$\begin{array}{c}\text { At least one abnormal Doppler finding of the } \\
\text { MCA during pregnancy, n (\%) }\end{array}$ & $2(22.2)$ & $44(10.3)$ & 0.244 \\
IUGR, n (\%) & $2(22.2)$ & $31(7.3)$ & 0.143 \\
\hline
\end{tabular}

2006-2015 508 MC neonates (twin s), 436 with complete ultrasound data, born after 175 days of gestation without IUD.

Table 5. Monochorionic placentation and pathology findings of multiple pregnancies in our institution between 1998 and 2015.

\begin{tabular}{cccc}
\hline Variable & NEC (n= 13) & No NEC (n=385) & $P$-value \\
\hline Umbilical cord insertion & & & \\
- Velamentous, n (\%) & $3(25.0)$ & $47(12.4)$ & 0.200 \\
- Marginal, n (\%) & $3(25.0)$ & $100(26.5)$ & 0.235 \\
- Paracentral, n (\%) & $5(41.7)$ & $218(57.7)$ & 0.910 \\
- Central, n (\%) & $1(8.3)$ & $13(3.4)$ & 0.270 \\
Placental weight, mean, grams (SD) & $241.0(64.5)$ & $344.9(135.6)$ & 0.009 \\
Unequal placental sharing, n (\%) & $10(76.9)$ & $243(60.6)$ & 0.235 \\
VV anastomoses, n (\%) & $3(23.1)$ & $93(24.2)$ & 0.929 \\
AV anastomoses, n (\%) & $13(100)$ & $346(90.3)$ & 0.239 \\
VA anastomoses, n (\%) & $7(53.8)$ & $291(76.0)$ & 0.069 \\
anastomoses, n (\%) & $10(76.9)^{\dagger}$ & $126(31.4)$ & 0.001 \\
AA anastomoses, n (\%) & $9(69.2)$ & $328(85.2)$ & 0.116 \\
\hline
\end{tabular}

1998-2015 770 MC neonates, 398 with placental pathology reports and born after 175 days of gestation without IUD. ${ }^{\dagger} 8$ of 10 neonates with NEC and unbalanced interfetal transfusion were the donor child.

Table 6. Characteristics of neonatal NEC cases 2006-2015.

\begin{tabular}{cccc}
\hline Variable & DC (n=26) & MC (n=11) & $P$-value \\
\hline Birth weight (g), mean (SD) & $1235.4(435.9)$ & $1029.8(447.8)$ & 0.217 \\
Gestational age at birth (days), mean (SD) & $200.4(15.8)$ & $206.4(29.2)$ & 0.430 \\
Bell stage 1, n (\%) & Bell's stages & & \\
Bell stage 2, n (\%) & $6(23.1)$ & $1(9.1)$ & 0.649 \\
Bell stage 3, n (\%) & $7(26.9)$ & $2(18.2)$ & 0.695 \\
& $13(50.0)$ & $8(72.7)$ & 0.285 \\
Presentation NEC (days after birth), mean (SD) & $10.7(6.7)$ & $15.2(14.1)$ & 0.190 \\
Rectal blood loss, n (\%) & $12(46.2)$ & $5(50.0)$ & 0.836 \\
Inotropic support, n (\%) & $8(30.8)$ & $3(30.0)$ & 0.964 \\
\hline
\end{tabular}


Continued

\begin{tabular}{|c|c|c|c|}
\hline Need for blood transfusion, $\mathrm{n}(\%)$ & $15(71.4)$ & $5(50.0)$ & 0.244 \\
\hline$\geq 7$ days antibiotic therapy, $\mathrm{n}(\%)$ & $16(61.5)$ & $8(80.0)$ & 0.357 \\
\hline \multicolumn{4}{|c|}{ Radiologic findings } \\
\hline Intestinal pneumatosis, n (\%) & $20(76.9)$ & $8(80.0)$ & 0.842 \\
\hline \multicolumn{4}{|c|}{ Surgery } \\
\hline Need for surgery, n (\%) & $12(46.2)$ & $8(72.7)$ & 0.138 \\
\hline $\begin{array}{c}\text { Need for surgery due to a } \\
\text { secondary intestinal stenosis, } \mathrm{n}(\%)\end{array}$ & $1(3.9)$ & $0(0)$ & 0.510 \\
\hline \multicolumn{4}{|c|}{ Pathology } \\
\hline Pathology findings confirming NEC, $\mathrm{n}(\%)$ & $4(36.4)$ & $1(50.0)$ & 0.715 \\
\hline
\end{tabular}

\section{Discussion}

To our knowledge, this is the first study investigating the pathophysiologic underpinnings of the relatively high prevalence of NEC in MC multiple pregnancies. We have shown that NEC is almost twice as common in MC twins as compared to DC multiples, a difference that remains after correction for known risk factors such as birthweight, gestational age at birth and nulliparity. There were no significant differences in clinical appearance or in biochemical or radiologic features, nor in treatment modalities of neonates with NEC following MC or DC multiple pregnancies. MC twins have a higher risk for sepsis, possible due to their prematurity and low birthweight. In MC twins, there were no differences in Doppler flow abnormalities between cases with and without NEC. Pathology findings of MC placentas showed that unbalanced interfetal transfusion through $\mathrm{AV}$-anastomoses between both placental territories occurred significantly more often in the NEC group.

Strengths of this study include the relatively large cohort size of multiple pregnancies, with extensive ultrasound measurements and detailed placental pathology reports. The main limitation is the retrospective design, which makes it hard to observe the pathophysiologic process because we can only observe the end markers, and the relatively large proportion of data of both ultrasound and pathology findings missing. In addition, due to the low incidence of NEC and monochorionicity, subgroup analyses contained small numbers, thereby reducing the likelihood of identifying significant associations.

A higher prevalence of NEC in MC twin pregnancies compared with DC multiple pregnancies has previously been reported by our group [9]. However, the exact pathophysiology of NEC remains unknown and is likely multifactorial, with ischemia of the intestine playing a significant role. With the current investigation, we were able to confirm the higher incidence of NEC in MC multiple pregnancies. The most relevant distinction between $\mathrm{MC}$ and DC placentas is the presence of vascular anastomoses in MC placentas, with the potential of interfetal transfusion that can lead to subtle hypoxic damage of the bowel. More specif- 
ically, the presence of unbalanced arterial-venous anastomoses (in number and/or in caliber) can cause unbalanced interfetal blood transfusion. Unbalanced interfetal transfusion through vascular anastomoses in the MC placenta could lead to a hemodynamic imbalance, potentially causing hypoperfusion of end organs such as the intestinal mucosa. This hypoperfusion could result in subtle hypoxic damage that increases the risk of developing NEC after birth. The potential association between bowel hypoperfusion and NEC has been described in neonates with hypoplastic left heart syndrome, coarctatio aortae and related abnormal abdominal aorta hemodynamics [12]. In this study, unbalanced interfetal transfusion was found significantly more often in MC multiples developing NEC, supporting our hypothesis. This finding was strengthened by the absence of large compensating AA-anastomoses in MC twins with NEC [13]. This is the first study that reports an association between unbalanced interfetal transfusion and the occurrence of NEC in MC multiple pregnancies.

A higher incidence of cerebral white matter lesions (WMLs) has been described in MC multiple pregnancies as compared to DC pregnancies in preterm neonates, most notably when MC pregnancies were complicated by discordant weight, TTTS or single intra-uterine death [14]. These findings are in line with the theory that relative hypoperfusion causes end organ hypoxic damage. One could then hypothesize that intraventricular hemorrhage is more common after MC multiple pregnancies, because of the ischemic encephalopathy underlying this disease. However, the current study as well as previous reports fails to demonstrate an association between MC multiple pregnancies and intraventricular hemorrhage, possibly due to fetal brain sparing that protects the brain from hypoxia [15].

Prenatal diagnosis of interfetal transfusion is difficult unless severely unbalanced interfetal transfusion occurs, resulting in the development of TTTS. In support of our hypothesis, NEC is seen more often after MC multiple pregnancies complicated by TTTS. Ultrasound examination in general does not detect the change in hemodynamics caused by vascular anastomoses. However, in the presence of large AA-anastomoses a typical Doppler pattern of intermittent abnormal flow can be seen [16]. Unfortunately, such patterns were not recorded in our database since this technique is quite recent and not yet generally introduced in common practice.

Concerning ultrasound data, we did not show an association between abnormal Doppler findings and the occurrence of NEC. Once could argue that relatively limited hypoperfusion is more prominent in the small arteries of end organs, which then cannot be detected by Doppler measurement of the umbilical artery. IUGR was more frequently seen in fetuses later developing NEC, although this finding was not significant. Others have shown an association between abnormal umbilical artery Doppler flow patterns and NEC in preterm infants, also when corrected for birthweight and gestational age [17]. Moreover, an association between IUGR and NEC has been described [18]. Although both in- 
vestigations were relatively small retrospective studies, they underline the potential role of IUGR related blood perfusion changes in the development of NEC. In case of placental insufficiency resulting in IUGR, redistribution occurs in order to spare critical organs (brain sparing effect). This may lead to a relative hypoperfusion of the intestines, thereby increasing the risk of NEC. The relation between vascular pathology (placental infarcts) and NEC is described before [19]. The possible association between Doppler abnormalities or IUGR and the development of NEC needs further research. Fetal Doppler assessment of the mesenteric artery may be of great interest, although an association between antenatal Doppler abnormalities of this artery and NEC has not been confirmed [20].

In case of MC multiples, the hypoxic damage to the intestines likely occurs during pregnancy. We therefore evaluated whether NEC developed sooner after birth, and whether the disease was more severe, in MC multiples as compared to DC twins. Unlike our hypothesis, NEC did not develop earlier in MC neonates than in DC neonates. A different feeding policy in both groups may mask a difference in time of onset: MC neonates are born significantly more often preterm and subsequently have lower birthweights compared with DC neonates. We did however find a significant difference in proportion of children that needed a blood transfusion directly after birth in the MC group with development of NEC. The relation between anemia, red blood cell transfusion and the development of NEC is unclear. Some studies suggest anemia or red blood cell transfusion is an independent risk factor for the development of NEC. Although, a recent bigger prospective trial does not show this relation, a higher incidence of NEC in children with anemia could support our theory: because of the subtle intra uterine hypovolemic state of the donor twins correlates with a lower red blood count and a higher incidence of NEC. However, further research about the cause and effect of anemia and NEC is needed.

Despite the similar distribution of Bell's stages among DC and MC multiples with NEC, suggesting no difference in severity between both groups, a trend towards a higher need for surgery was seen in the MC group. This difference may suggest a higher rate of irreversible ischemic damage in MC twins as compared to DC twins. It is tempting to speculate that an intra-uterine ischemic incident has rendered the intestines more prone to severe damage in MC multiples. The fact that we did not find any differences in Bell's classification between both groups may be due to the inclusion of a broad and heterogeneous series of neonatal intestinal disease using this classification, although the criteria are clear and give a graduation of severity. It is possible that the heterogeneous and multifactorial etiology of NEC makes it difficult to retrospectively determine the clinical role of subtle hypoxic damage to the bowel [5] [21].

\section{Conclusion}

NEC is more common after monochorionic multiple pregnancies and is associated with unbalanced interfetal transfusion through arterial-venous anastomoses. Most likely, subtle ischemic damage due to intra-uterine fetal hypoperfu- 
sion plays an important role in the development of NEC in this group, although it is still unclear which factor is of decisive importance in the development of NEC. Further research, a prospective study, is needed to determine the possible association between monochorionic placental abnormalities and the course of NEC, and related treatment strategies. In addition, the role of intra-uterine subtle hypoxic end-organ damage in the development of NEC in singleton pregnancies remains to be determined and warrants further research.

\section{Acknowledgements}

We thank J. Groeneweg for comments that greatly improved the manuscript.

\section{Contribution to Authorship}

All authors have contributed significantly.

\section{Conflicts of Interest}

None of the authors have any competing interest.

\section{References}

[1] Guthrie, S.O., Gordon, P.V., Thomas, V., Peabody, J. and Clark, R.H. (2003) Necrotizing Enterocolitis among Neonates in the United States. Journal of Perinatology, 23, 278-285. https://doi.org/10.1038/sj.jp.7210892

[2] Kosloske, A.M. (1994) Epidemiology of Necrotizing Enterocolitis. Acta Paediatrica, 396, 2-7. https://doi.org/10.1111/j.1651-2227.1994.tb13232.x

[3] Yee, W.H., Soraisham, A.S., Shah, V.S., Aziz, K., Yoon, W., Lee, S.K., et al. (2012) Incidence and Timing of Presentation of Necrotizing Enterocolitis in Preterm Infants. Pediatrics, 129, 298-304. https://doi.org/10.1542/peds.2011-2022

[4] Sharma, R. and Hudak, M.L. (2013) A Clinical Perspective of Necrotizing Enterocolitis: Past, Present, and Future. Clinics in Perinatology, 40, 27-51. https://doi.org/10.1016/j.clp.2012.12.012

[5] Neu, J. (2014) Necrotizing Enterocolitis: The Mystery Goes On. Neonatology, 106, 289-295. https://doi.org/10.1159/000365130

[6] Huda, S., Chaudhery, S., Ibrahim, H. and Pramanik, A. (2014) Neonatal Necrotizing Enterocolitis: Clinical Challenges, Pathophysiology and Management. Pathophysiology, 21, 3-12.

[7] Lin, P.W. and Stoll, B.J. (2006) Necrotizing Enterocolitis. The Lancet, 368, 1271-1283. https://doi.org/10.1016/S0140-6736(06)69525-1

[8] Burjonrappa, S.C., Shea, B. and Goorah, B. (2014) NEC in Twin Pregnancies: Incidence and Outcomes. Journal of Neonatal Surgery, 20, 45.

[9] Hack, K.E.A., Derks, J.B., Elias, S.G., Franx, A., Roos, E.J., Voerman, S.K., et al. (2008) Increased Perinatal Mortality and Morbidity in Monochorionic versus Dichorionic Twin Pregnancies: Clinical Implications of a Large Dutch Cohort Study. BJOG: An International Journal of Obstetrics \& Gynaecology, 115, 58-67. https://doi.org/10.1111/j.1471-0528.2007.01556.x

[10] Bell, M.J., Ternberg, J.L., Feigin, R.D., Keating, J.P., Marshall, R., Barton, L., et al. (1978) Neonatal Necrotizing Enterocolitis: Therapeutic Decisions Based upon Clin- 
ical Staging. Annals of Surgery, 187, 1-6. https://doi.org/10.1097/00000658-197801000-00001

[11] Hack, K.E., van Gemert, M.J., Lopriore, E., Schaap, A.H., Eggink, A.J., Elias, S.G., et al. (2009) Placental Characteristics of Monoamniotic Twin Pregnancies in Relation to Perinatal Outcome. Placenta, 30, 62-65. https://doi.org/10.1016/j.placenta.2008.09.016

[12] Miller, T.H., Minich, L.L., Lambert, L.M., Joss-Moore, L. and Puchalski, M.D. (2014) Abnormal Abdominal Aorta Hemodynamics Are Associated with Necrotizing Enterocolitis in Infants with Hypoplastic Left Heart Syndrome. Pediatric Cardiology, 35, 616-621. https://doi.org/10.1007/s00246-013-0828-8

[13] Villiers, S.F., Slaghekke, F., Middeldorp, J.M., Walther, F.J., Oepkes, D. and Lopriore, E. (2012) Arterio-Arterial Vascular Anastomoses in Monochorionic Placentas with and without Twin-Twin Transfusion Syndrome. Placenta, 33, 652-654. https://doi.org/10.1016/j.placenta.2012.05.003

[14] Adegbite, A.L., Castille, S., Ward, S. and Bajoria, R. (2005) Prevalence of Cranial Scan Abnormalities in Preterm Twins in Relation to Chorionicity and Discordant Birth Weight. European Journal of Obstetrics and Gynecology, 119, 47-55. https://doi.org/10.1016/j.ejogrb.2004.06.016

[15] Giussani, D.A. (2016) The Fetal Brain Sparing Response to Hypoxia: Physiological Mechanisms. The Journal of Physiology, 594, 1215-1230. https://doi.org/10.1113/JP271099

[16] Gratacós, E., Lewi, L., Carreras, E., Becker, J., Higueras, T., Deprest, J., et al. (2004) Incidence and Characteristics of Umbilical Artery Intermittent Absent and/or Reversed End-Diastolic Flow in Complicated and Uncomplicated Monochorionic Twin Pregnancies. Ultrasound in Obstetrics \& Gynecology, 23, 456-460. https://doi.org/10.1002/uog.1013

[17] Kamoji, V.M., Dorling, J.S., Manktelow, B., Draper, E.S. and Field, D.J. (2008) Antenatal Umbilical Doppler Abnormalities: An Independent Risk Factor for Early Onset Neonatal Necrotizing Enterocolitis in Premature Infants. Acta Paediatrica, 97, 327-331. https://doi.org/10.1111/j.1651-2227.2008.00671.x

[18] Aucott, S.W., Daonohui, P.K. and Northington, F.J. (2004) Increased Morbidity in Severe Early Intrauterine Growth Restriction. Journal of Perinatology, 24, 435-440. https://doi.org/10.1038/sj.jp.7211116

[19] Moore, S.W., Arnold, M. and Wright, C. (2013) Necrotizing Enterocolitis and the Placenta-A Key Etiological Link. Journal of Pediatric Surgery, 48, 359-362. https://doi.org/10.1016/j.jpedsurg.2012.11.020

[20] Deepak, L., Mukhapadhyay, K., Sodhi, K.S., Jain, V. and Kumar, P. (2013) Superior Mesenteric Artery Doppler Is Poor at Predicting Feed Intolerance and NEC in Preterm Small for Gestational Age Neonates. The Journal of Maternal-Fetal \& Neonatal Medicine, 26, 1855-1859. https://doi.org/10.3109/14767058.2013.799649

[21] Neu, J. and Walker, W.A. (2011) Necrotizing Enterocolitis. The New England Journal of Medicine, 364, 255-264. https://doi.org/10.1056/NEJMra1005408 


\section{Key Message}

Necrotizing enterocolitis (NEC) is a serious illness of the neonate with ischemic necrosis of the intestinal mucosa. This research shows that NEC occurs more commonly in monochorionic twins as compared to dichorionic twins and is associated with unbalanced interfetal transfusion through placental vascular anas-

tomoses, that most likely cause intra-uterine hypotension and subtle end organ hypoxic damage.

\section{Abbreviations}

NEC: necrotizing enterocolitis

DC: dichorionic

MC: monochorionic

NICU: neonatal intensive care unit

TTTS: twin-to-twin transfusion syndrome

IUGR: intra-uterine growth restriction

IVH: intraventricular hemorrhage

AA: arterial-arterial

VV: venous-venous

$\mathrm{AV}$ : arterial-venous

VA: venous-arterial 